\title{
Occlusion-Aware Menu Design for Digital Tabletops
}

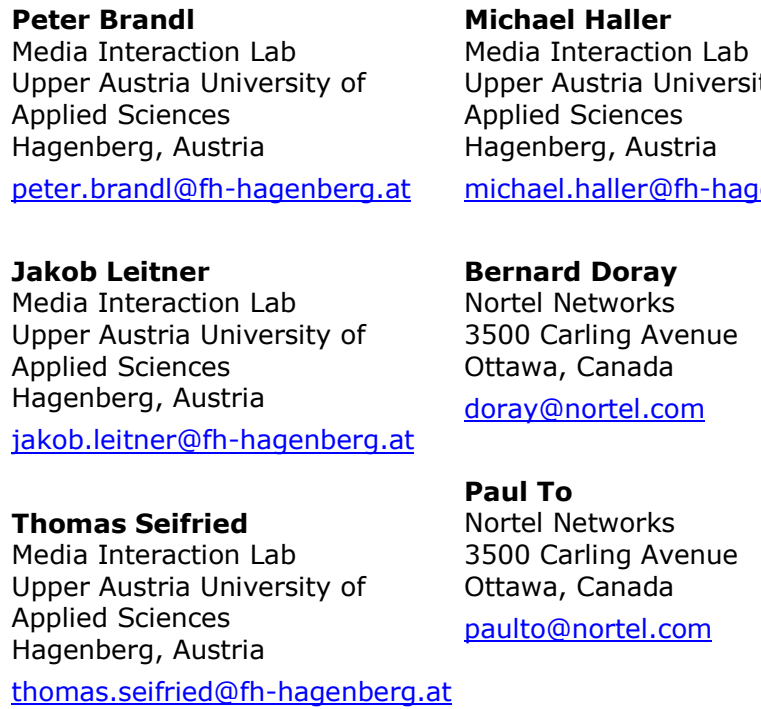

\author{
Abstract \\ In this paper, we describe the design of menus for \\ multi-user digital tabletops. On direct input surfaces, \\ occlusions created by the user's hand decrease \\ interaction performance with menus. The key design \\ criteria are to avoid these occlusions and to adapt the \\ menu placement to the user's handedness and position \\ on the tabletop. We present an adaptive menu \\ placement method based on direct touch and pen \\ tracking that allows correct menu placement around the \\ table. As an extension, we propose adding a gesture \\ input area for fast interaction which can be partly \\ occluded by the user's hand.
}

\section{Keywords}

Tabletop, Digital Whiteboard, Pie Menu, Pen-based Interface, Ergonomic Evaluation.

\section{ACM Classification Keywords}

H.5.2 [Information Interfaces and Presentation]: User Interfaces - Input devices and strategies, Interaction styles, Evaluation/methodology.

\section{Introduction}

Interaction with large direct digital surfaces is strongly influenced by physical restrictions. Reachability of items or occlusions through the user's body require novel 
design considerations for appropriate interfaces [9]. As Apitz et al. [1] noticed for example, traditional menus are not very well adapted to direct pen interaction. Menus that appear on the location where they are activated seem to be a better choice for large interactive surfaces, where the input is normally done with a pen or a direct finger touch (cf. figure 1).

Direct input on digital tabletops is also affected by the handedness and the position of the user. Hancock et al. [5] studied selection times for pop-up menus with pen input and noticed that adapting to the user's handedness is necessary. Otherwise, either a left or right-handed user will be discriminated, depending on the application settings. In their study, the authors noticed a slower performance for occluded areas. These are mirrored for left and right-handed users. This observation shows that occlusion is strongly related to handedness and hand posture. Moreover, the study showed that not occluded menus are better accepted by the users and can enhance performance.

In this paper, we present our observations on occlusions for horizontal surfaces. Based on that, we propose a new design for a tabletop menu that avoids occlusions created by the user's hand. By using partly occluded areas for gesture input, we extend the functionality of a traditional point-and-click menu. To account for the handedness of users we apply an adaptive menu placement method based on direct touch and pen tracking. In addition, we show that our flexible system also works for multi-user tabletop setups.

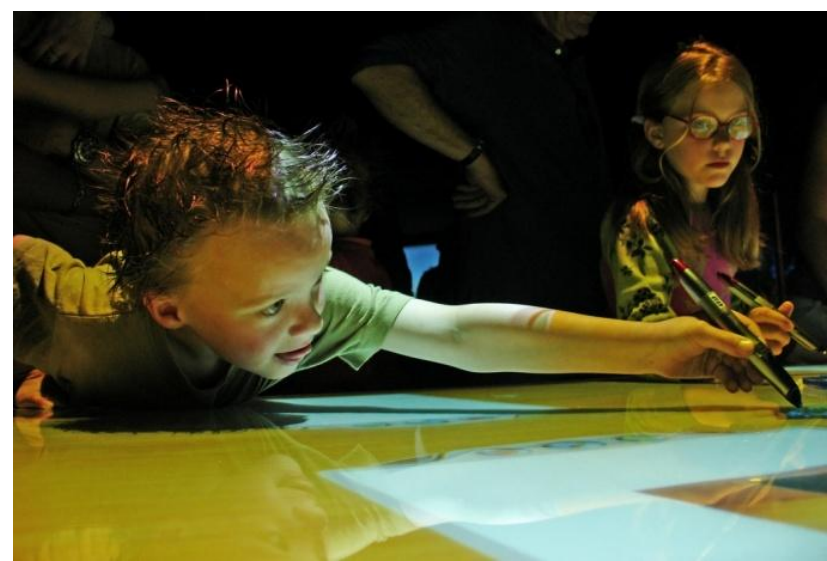

figure 1: Menu placement and reachability play an important role on digital tabletops.

\section{Related Work}

Screen Occlusion

Occlusion of direct input surfaces has been investigated on small mobile devices like PDAs and on tabletops.

Bieber et al. [2], for example, explored screen coverage for pen interaction and touch screens. They presented an analytic approach to measure the covering of touch screen areas and interaction elements. They mentioned that the differences between left and right-handed users have an effect on screen coverage, but for their analysis, they assume that the average user is right-handed.

Leithinger et al. [9] investigated six different menu layouts for interactive tables under various cluttering conditions. In their study, they found out that menu types suffering from occlusion (pie menus for example) showed significant disadvantages compared to their proposed user-drawn context menus. 
Our approach contributes to these works by describing an occlusion avoiding menu design. We furthermore show a possibility to use partly occluded areas for gesture input.

\section{User Handedness Detection}

There have been previous research projects dealing with the automatic detection of the user's handedness. They are based on different input devices and assumptions about the user behavior. Kurtenbach et al. [8] present a method of automatically determining handedness of users for a bimanual drawing application that utilizes a stylus in one hand and a puck in the other.

Hancock et al. [5] suggest three different approaches to detect the user's handedness and according menu placements for one-handed pen interaction. They discuss a simple heuristic approach, a neural network and a Bayesian network model. Harrison et al. [6] explored annotation of digital documents on a handheld computer where they determined the handedness via pressure sensitive pads placed on the back of the device.

Our approach for adapting to the user's handedness is suitable for one-handed pen input and does not require any pre-defined settings. In contrast to Hancock's network models, our method does not need a learning phase of the system.

\section{Occlusion Observation}

In order to be able to design a menu that avoids occlusions, we invited 18 participants and observed differences of occlusions for left and right-handed users on horizontal surfaces (3 left-handed,15 right-handed). figure 2 shows the results of our observation. The mirror effect of occlusions for left and right-handed participants is clearly visible.

The average number of visible segments was 11.70 $(\mathrm{SD}=0.99)$ out of 16 segments for right-handed users and $11.17(\mathrm{SD}=0.56)$ for left-handed users. This mirrored pattern for left and right-handed users was also noticed by Hancock et al. [5].

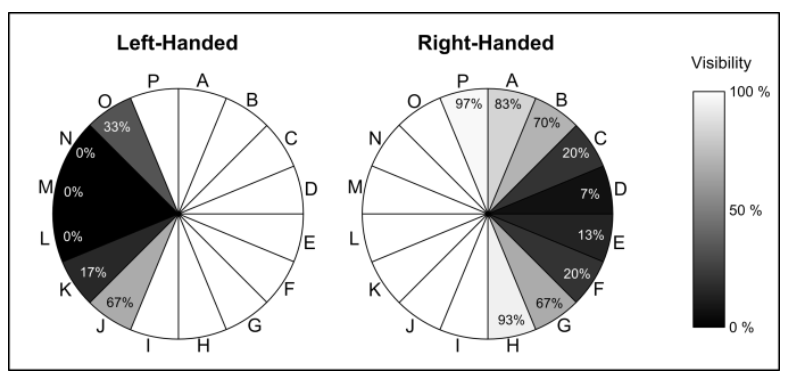

figure 2: The visibility of each segment for left-handed and right-handed users shows a mirror effect.

These results led us to the design of a menu with two specific features: First, we avoid placing items in occluded areas, thus improving the interaction with the menu. Second, we propose a method for adaptive menu placement on tabletops that solves the problem of left and right handedness.

\section{Prototype}

Based on our observations of occlusions we developed a menu for tabletops with direct pen input that is always visible to the user. The visibility of the menu is mainly influenced by the occlusion caused by the user's hand. Referring to a full $360^{\circ}$ circle of possible item 

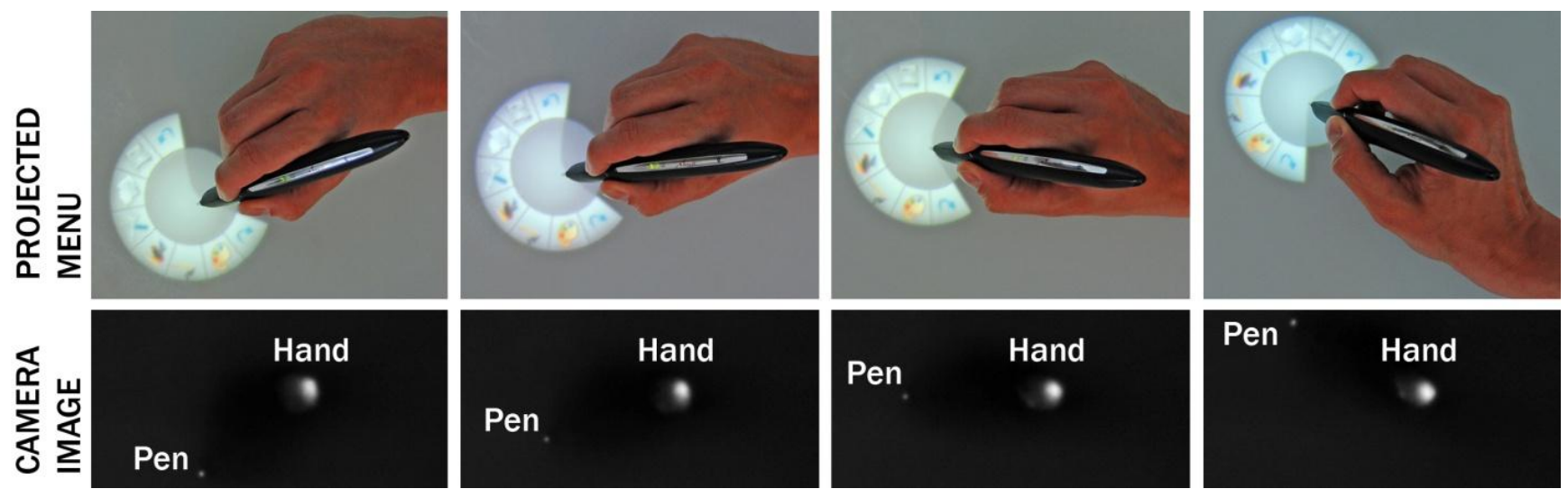

figure 3: Adaptive menu placement through a combination of FTIR multi-touch and Anoto pen tracking. The direction vector from the hand to the pen is used to determine the correct orientation.

placements around an invocation point, we found that $92^{\circ}$ of the circle are occluded on average.

\section{Menu Design}

According to this result, we designed a menu with items placed only in areas that are not occluded by the hand. Our design is inspired by the layout of circular menus [7]. The position of the menu is centered at the point of activation (cf. figure 4 ).
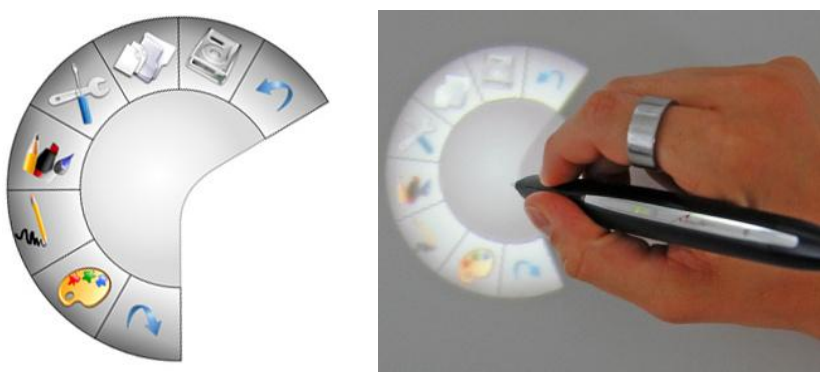

figure 4: Design of a menu that avoids occlusions caused by the user's hand.
Frequently used items are placed in the menu according to Hancock's results [5]. He reports that the movement along the "top-left to bottom-right axis is fastest for left-handed users" and the mirrored movement along the "top-right to bottom-left axis for right-handed users". Therefore we placed the undo buttons in those fast access positions in our design.

\section{Adaptive Menu Placement}

The proposed design as shown in figure 4 would only work for right-handed users and it would require a distinct orientation of the hand to ensure full visibility of all items. There are generally two different ways to overcome these restrictions: The first method is to adapt the placement to the user's preferences which he/she defines in advance. This method is not suitable for direct tabletop interaction, because we have to deal with different user positions around the surface. The other option is an adaptive menu placement which automatically adjusts to the user's current position. There are different methods for an adaptive solution [8][5][6]. We use a combination of FTIR multi-touch 
tracking [4] and Anoto pen tracking [3] to determine the user's hand position and the current pen position.

We observed that users tend to rest their hand on the surface when using direct pen input on tabletops due to fatigue effects. This behavior has also been noticed in previous research projects [5]. If we know the position where the users rest their hand and the position where they want to activate the menu, we can easily

determine the correct placement for the menu.

In our current setup, we assume that the hand is in contact with the surface when the user activates the menu. To obtain the correct orientation of the menu, we simply use the direction vector from the hand to the pen tip. The menu is centered on the pen's position and rotated with the information of the direction vector. figure 3 shows four different examples for a right-

handed user. This procedure provides two advantages: First, we have an automatic adaption for left and righthanded users, as the menu rotates according to the direction vector from hand to pen. Second, the orientation is correct from any perspective on the tabletop and occlusions are avoided.

We demonstrate only one possible solution that shows how adaptive menu placement in combination with our occlusion avoiding menu design works. Other methods like shadow tracking would provide a more general solution that also works if the users do not rest their hand on the surface [2].

\section{Multi-User Scenario}

Our system identifies the user through the ID that every Anoto pen delivers and assigns one menu to each of them. Hence we can support interaction of multiple users simultaneously. Due to the adaptive placement, the menu will be oriented towards the user independent of its position (cf. figure 5).

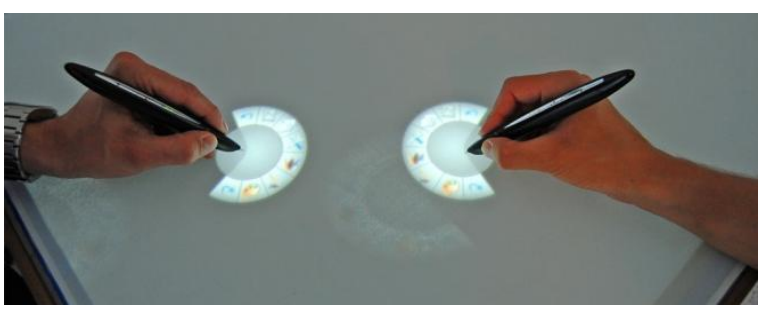

figure 5: The system supports multiple menus adapting to each user's position and handedness.

Point-and-Click Area vs. Gesture Area

We propose to use the occluded area as part of an interactive area for gesture input inside the menu (cf. figure 6 ). Our observations showed that occlusions are not a problem in this case if the area can be recognized and the user knows where he can start a gesture and which gestures he can use. The outer region of the menu should be used for the items which can be accessed with a simple point-and-click.

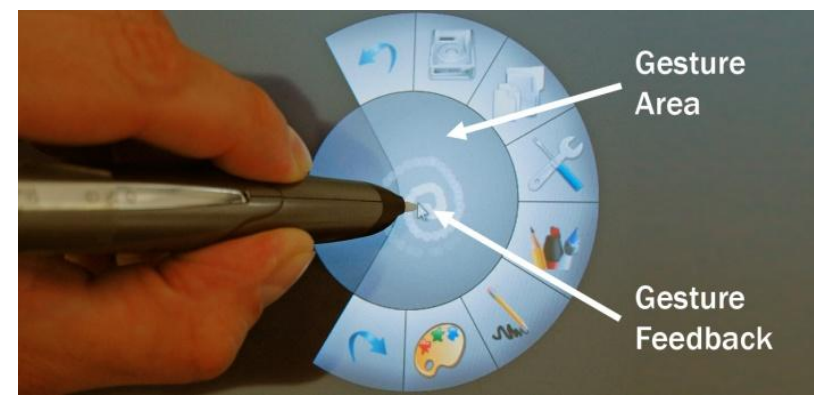

figure 6: Users can perform gestures on the circular gesture area inside the menu. 


\section{Conclusion \& Future Work}

In this paper, we have shown a menu design for digital tabletops with two main characteristics: The menu has an open side where no items are placed which avoids occlusions caused by the user's hand. Moreover, we presented a method for adaptive menu placement that automatically adapts to the user's handedness and position around the tabletop. Based on our multi-touch and pen tracking solution, we can also support multiuser scenarios with user identification.

As a next step, we are going to integrate this menu into our tabletop brainstorming application prototype for testing it in a real scenario. We will collect user feedback on our menu design and gesture interaction techniques. Our current menu shows 8 items in the point-and-click area. According to the suggested 8 items in a circular menu [7], we plan to test the ideal number of items for our design. We are interested in a solution for a digital whiteboard menu design where we expect some similarities to the tabletop version but also some substantial differences.

\section{References}

[1] Apitz, G. and Guimbretière, F. 2004. CrossY: a crossing-based drawing application. In Proc. of UIST '04, 3-12.

[2] Bieber, G., Abd Al Rahman, E.; Urban, B. Screen Coverage: A Pen-Interaction Problem for PDAs and
Touch Screen Computers. In Proc. of ICWMC '07, 87.

[3] Brandl, P., Haller, M., Hurnaus, M., Lugmayr, V., Oberngruber, J., Oster, C., Schafleitner, C., Billinghurst, M., 2007. An Adaptable Rear-Projection Screen Using Digital Pens And Hand Gestures. In Proc. of ICAT '07, 49-54.

[4] Han, J. Y. 2005. Low-cost multi-touch sensing through frustrated total internal reflection. Proc. of UIST '05, 115-118.

[5] Hancock, M. S. and Booth, K. S.: Improving Menu Placement Strategies for Pen Input. In Proc. of GI '04, 221-230.

[6] Harrison, B. L., Fishkin K. P., Gujar A., Mochon C., Want R., 1998. Squeeze me, hold me, tilt me! An exploration of manipulative user interfaces. In Proc. of $\mathrm{CHI}$ '98, 17-24.

[7] Hopkins, D. The design and implementation of pie menus, Dr. Dobb's Journal, 16.12 (Dec.1991):1626.

[8] Kurtenbach, G., Fitzmaurice, G., Baudel, T., and Buxton, W , 1997. The design of a GUI paradigm based on tablets, two-hands, and transparency. In Proc. of $\mathrm{CHI}, 35-42$.

[9] Leithinger, D. and Haller M., 2007. Improving Menu Interaction for Cluttered Tabletop Setups with UserDrawn Path Menus. In Proc. of TABLETOP '07, 121128. 